\title{
Etkin EEG Özellikleri Çıkarılarak Arousal Tespiti
}

\author{
Gizemnur Erol ${ }^{1}$, Fatma Zehra Göğüş ${ }^{2 *}$,Gülay Tezel ${ }^{3}$ \\ ${ }^{1}$ Konya Teknik Üniversitesi Bilgisayar Mühendisliği Bölümü, Konya, Türkiye \\ ${ }^{2}$ Konya Teknik Üniversitesi Bilgisayar Mühendisliği Bölümü, Konya , Türkiye (ORCID: 0000-0001-5035-7575) \\ ${ }^{3}$ Konya Teknik Üniversitesi Bilgisayar Mühendisliği Bölümü, Konya , Türkiye (ORCID: 0000-0003-1698-0106)
}

( $1^{\text {st }}$ International Conference on Computer, Electrical and Electronic Sciences ICCEES 2020 - 8-10 Ekim 2020)

(DOI: $10.31590 /$ ejosat.802946)

ATIF/REFERENCE: Erol, G., Göğüs, F. Z., \& Tezel, G. (2020). Etkin EEG Özellikleri Çıkarılarak Arousal Tespiti. Avrupa Bilim ve Teknoloji Dergisi, (Özel Sayı), 117-122.

\section{$\ddot{O} \mathbf{z}$}

Son zamanlarda toplumun en önemli problemlerinden biri olan uyku bozuklukları, bireylerin sağlı̆̆ını ve yaşam kalitesini ciddi şekilde etkilemektedir. Uykusuzluk (Insomnia), narkolepsi, uyku apnesi ve huzursuz bacak sendromu gibi birçok uyku bozukluklarının neden olduğu rahatsızlıklar vardır. Uyku bozukluklarına sebep olan ana faktör ise bireyin uyku anındaki uyanma ile sonuçlanamayan, uyku kalitesini düşüren uyku kesintileridir. Arousal diğer bir adı ile uyanayazma geçici olan bu kesintilerdir ve bir beyin dalga (Elektroansefalogram -EEG) aktivitesinin paternindeki ani değişikliği temsil etmektedir. Arousal tespiti genellikle EEG verileri kullanılarak Amerikan Uyku Tıbbı Akademisi (American Academy of Sleep Medicine-AASM) tarafindan belirlenen kriterlere göre yapılmaktadır. Bu çalışmada amaç, AASM tarafindan belirlenen kriterler doğrultusunda EEG sinyalleri vasıtasıyla hasta bireylerdeki arousalların tespitidir. Bu amaç doğrultusunda, öncelikle, çalışmaya dahil edilen 5 hasta bireyin tek kanallı (C3/A2) EEG sinyallerine sırasıyla filtreleme, normalizasyon ve segmantasyon önişlemleri uygulanmıştır. Daha sonra Spektral Güç Yoğunluğu (Power Spectral Density-PSD) ve Ayrık Dalgacık Dönüşümü (Discrete Wavelet Transform-DWT) yöntemleri ile gerçekleştirilen özellik çıkarma süreci sayesinde, EEG sinyal segmentlerine ait 2 özellik seti ve bu özellik setlerinin birleş̧irilmesiyle 3. özellik seti oluşturulmuştur. Ardından oluşturulan 3 özellik seti üzerine Sarmal Alt Küme Değerlendirme (Wrapper Subset Evaluation-WSE) özellik seçme yöntemi uygulanarak etkin özellikler belirlenmiştir. Nihai olarak belirlenen özelliklerin Yapay Sinir Ağları (YSA) ve Rasgele Orman (RO) algoritmaları tarafından sınıflandırılmaları ile arousal içeren EEG segmentleri tespit edilmiştir. Gerçekleştirilen bu çalışmaların beraberinde EEG sinyal kayıtlarından başka hiçbir PSG sinyal kaydına ihtiyaç duymadan, yalnızca tek kanallı EEG sinyalleri ile oldukça başarılı sonuçlar elde edildiği tespit edilmiştir. Çalı̧̧ma sonucunda ise Özellik Seti 3'ün etkin özellikleri ve YSA ile en yüksek doğruluk oranı \%99.05 olarak elde edilmiştir.

Anahtar Kelimeler: Arousal, EEG, Spektral Güç Yoğunluğu, Ayrık Dalgacık Dönüşümü, Sarmal Alt Küme Değerlendirme, Yapay Sinir Ağları, Rasgele Orman Algoritması

\section{Arousal Detection by Extracting Efficient EEG Features}

\begin{abstract}
Nowadays, sleep disorders, one of the most important problems of society, affect the health and quality of life of individuals seriously. There are diseases caused by many sleep disorders such as insomnia, narcolepsy, sleep apnea and restless legs syndrome. The main factor that causes sleep disorders is sleep interruptions that don't result in the awakening of the individual during sleep and reduce sleep quality. Arousal, also known as awakening, are these temporary interruptions and represent a sudden change in the pattern of brainwave (Electroencephalogram-EEG) activity. Generally, Arousal detection is performed by utilizing EEG datas according to the criterions determined by American Academy of Sleep Medicine (AASM). This study's purpose is to detect arousals of patients through EEG, in accordance with the criterions of ASSM. For this purpose, primarily, filtering, normalization and segmentation preprocesses were applied to single channel (C3 / A2) EEG signals of 5 patients included in the study, respectively. Afterwards 2 feature sets and 3rd feature set by combining these 2 feature sets were generated for EEG signal segments thanks to feature extraction processing carried out Power Spectral Density (PSD) and Discrete Wavelet Transform (DWT) methods. Subsquently, efficient
\end{abstract}

\footnotetext{
* Sorumlu Yazar: Konya Teknik Üniversitesi, Mühendislik ve Doğa Bilimleri Fakültesi, Bilgisayar Mühendisliği Bölümü, Konya, Türkiye, ORCID: 0000-0001-5035-7575, fzgogus@ktun.edu.tr
} 
features were determined by implementing Wrapper Subset Evaluation (WSE) that is feature selection method on to the 3 features sets created. Finally, the EEG segments containing arousals were detected by classifying the determined features by Artificial Neural Networks (ANN) and Random Forest (RO) algorithms. With these studies performed, it has been determined to achieve successful results with only single-channel EEG signals without the need for any PSG signal recording other than EEG signal recording. As a result of the study, the highest accuracy rate (99.05\%) were obtained by using the effective features of Feature Set 3 and ANN.

Keywords: Arousal, EEG, Spectral Power Density, Discrete Wavelet Transform, Wrapper Subset Evaluation, Artificial Neural Networks, Random Forest Algorithm

\section{Giriş}

Polisomnografi (PSG) uyku bozuklukları tanısında kullanılan, hasta üzerine takılan elektrotlar vasıtasıyla gece uykusu boyunca hastanın solunum faaliyeti, beyin dalgaları (Elektroansefalogram-EEG), göz hareketleri (Elektrookülogram-EOG), kas aktivitesi (Elektromiyogram-EMG) vb. sinyallerin belli periyotlarla, eş zamanlı ve devamlı olarak kaydedilmesi ile yapılan bir incelemedir.

Arousal (uyanayazma) derin uykudan yüzeysel uyku evresine ya da uyanıklık durumuna kısa süreli ani geçişlerdir. Arousal sonucunda, uyku kalitesini düşüren ve kişilerin ertesi gün hatırlayamayacakları uyku bölünmeleri meydana gelir. Bu bölünmeler nedeniyle kişilerde aşırı uykusuzluk sorunu ortaya çıkar (Demir ve ark., 2012). Oluşan uykusuzluk kişilerin öğrenme becerilerini ve dikkatlerini azaltır, hafızalarını ve konsantrasyonlarını zayıflatır ve bunların sonucunda günlük ve iş yaşamlarında problem yaşamalarına sebebiyet verir (Demir ve ark., 2012). Bu nedenle arousalın tespit edilebilmesi oldukça önem arz etmektedir.

Arousal tespiti PSG sinyallerinden olan EEG, EMG ve EOG sinyalleri kullanılarak gerçekleştirilir. Bu kayıtlardan en sık tercih edileni ise EEG'dir (Wallant ve ark., 2016; Varela, Pereira, Estévez ve Bonillo, 2017). Amerikan Uyku Tıbbı Akademisi (American Academy of Sleep Medicine - AASM), bir EEG arousalı şu şekilde tanımlar: Alfa, teta ve/veya $16 \mathrm{~Hz}$ 'den daha büyük frekanslar içeren EEG'deki ani frekans değişimleridir (AASM, 2012). AASM'ye göre meydana gelen bu frekans değişikliğinin arousal olarak skorlanabilmesi için ise en az 3 saniye ve daha uzun süreli olması gerekmektedir (AASM, 2012).

$\mathrm{Bu}$ çalışmada, bu kurallar doğrultusunda, tek kanallı EEG sinyalleri kullanılarak arousal tespitinin gerçekleştirilmesi amaçlanmıştır. Bu kapsamda, EEG sinyallerine sırasıyla filtreleme, normalizasyon ve segmentasyon işlemleri uygulanmış ve sinyalleri karakterize eden özellikler çeşitli özellik çıkarma yöntemleri ile elde edilmiştir. Ardından, Weka (Witten ve ark., 1999) platformu kullanılarak segmente edilen EEG sinyallerindeki arousal içeren segmentler Rastgele Orman (RO) ve Yapay Sinir Ağları (YSA) algoritmaları ile tespit edilmiştir.

\section{Materyal ve Metot}

\subsection{Veri Seti}

Bu çalışma kapsamında arousal problemi yaşayan 5 hasta bireye ait tek kanal (C3/A2) EEG sinyalleri kullanılmıştır. 1 hastanın EEG sinyali PhysioNet'de bulunan The Sleep Heart Health Study (SHHS) çalışmasından elde edilmiştir (Goldberger ve ark., 2000). Bu EEG sinyali açık erişimlidir ve sinyalin örnekleme frekansı $125 \mathrm{~Hz}$ 'dir. Diğerleri ise Göğüş ve ark.'nın 2020 yılında yapmış oldukları çalışmadaki, gerekli etik kurul raporu ve hasta rızaları alınarak gerçekleştirilen 4 hastaya ait PSG kayıtlarının $200 \mathrm{~Hz}$ örnekleme frekanslı EEG sinyalleridir (Göğüş ve ark., 2020).

\subsection{Filtreleme ve Normalizasyon}

Sinyaller ortam seslerinden, kayıt cihazlarının takılıp çıkarılması gibi birçok olaydan kaynaklı gürültüler içerebilir. Bu gürültüler, sinyal çalışmalarında verimli sonuç almayı engellediği için sinyaller gürültülerden arındırılmalıdır. Sinyallerin gürültülerden arındırılması amacıyla çalışmaya dahil edilen 5 hastaya ait EEG sinyalleri frekans aralı̆̆ $0.3-35 \mathrm{~Hz}$ olan 'Bandpass' tipi 3. dereceden Butterworth Filtresi ile filtrelenmiştir (Wallant ve ark., 2016). Bu işlemin ardından, filtre edilmiş sinyaller -1 ile 1 aralığında normalize edilmiştir. Gerçekleştirilen filtreleme ve normalizasyon işlemleri sonucundaki elde edilen sinyaller Şekil 1'de gösterilmektedir.

\subsection{Segmentasyon}

Sinyal çalışmalarında sinyaller, belirli sürelerdeki epoklara bölünerek bu epoklar (segment) üzerinde çalışılır. AASM'nin (AASM, 2012) arousal için belirttiği, bir frekans değişikliğinin arousal olarak skorlanmast için en az 3 saniye ve daha uzun süre olmalı, kuralı vardır. Yani bir arousal minimum 3 saniye olmalıdır. Bu nedenle bu çalışmada bir segment boyutu da 3 saniye olarak belirlenmiştir. Arousal tespiti için yapılan segmentlemenin örtüşmesiz olması veri kaybına yol açarak arousal noktalarını kaçırmaya neden olabilir. Bu nedenle bu çalı̧̧mada Şekil 2'de görüldüğü gibi 1 saniye kaydırmalı, 2 saniye örtüşmeli pencereleme işlemi gerçekleştirilerek EEG sinyalleri segmente edilmiştir. 
a)

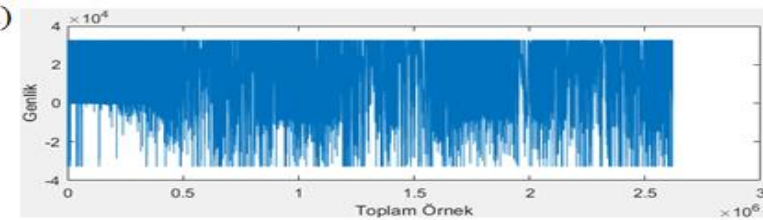

b)

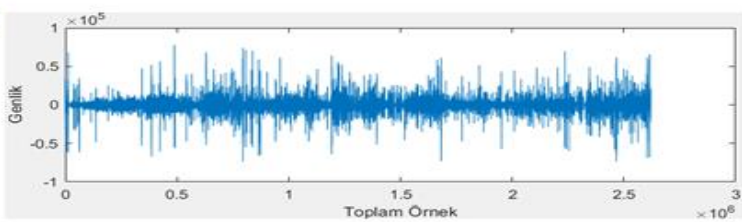

c)

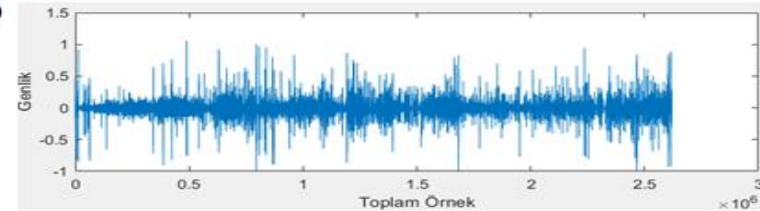

Şekil 1. (a) Ham EEG, (b) Filtre Edilmiş EEG, (c) Normalize Edilmiş EEG

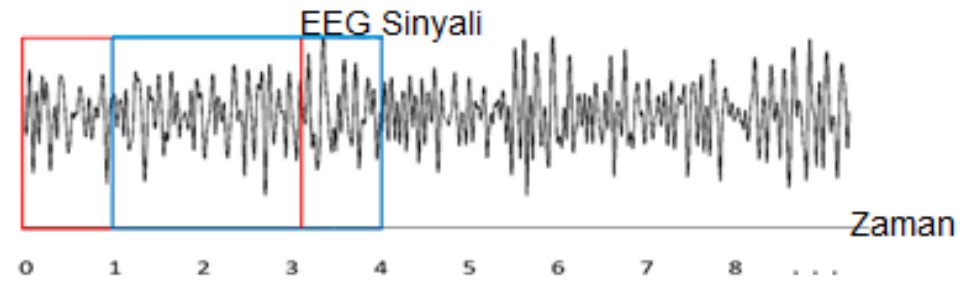

Şekil 2. EEG Sinyallerinin Segmentasyonu

\subsection{EEG Sinyal Zaman ve Frekans Analizi}

AASM'ye (AASM, 2012) göre arousal tanımı, EEG'de alfa, teta ve/veya 16 Hz'den daha büyük frekanslar içeren dalgalardır. Yani arousal tespiti belirli özel bantların seçilerek onlar üzerinde işlemler yapılmasını gerekmektedir. Bu çalışmada arousal tespiti için kullanılan özel bantlar alfa, teta, delta, sigma ve >16 Hz. olarak belirlenmiştir (Varela ve ark., 2017). Bu özel bantların frekans aralığı Tablo 1 'de verilmiştir.

Tablo 1. EEG Frekans Bantlart

\begin{tabular}{l|l}
\hline Bant & Frekans Aralı̆̆ $\mathbf{( H z )}$ \\
\hline$\Delta$ (Delta) & $0.5-4$ \\
\hline$\Theta($ Teta $)$ & $4-7$ \\
\hline$\alpha($ Alfa $)$ & $8-12$ \\
\hline$\sigma($ Sigma $)$ & $12-15$ \\
\hline$>16$ & $>16$ \\
\hline
\end{tabular}

\subsubsection{Spektral Güç Yoğunluğu ile Özellik Çıkarma}

EEG işaretleri, beynin sinirsel faaliyeti sonucu elde edilen biyoelektriksel işaretlerdir ve bu işaretlerde beynin fonksiyonları ile ilgili birçok bilgi saklanmaktadır. Bu nedenle, EEG sinyallerinin frekans bileşenleri çokça önem arz etmektedir. Patolojik belirtilerin bulunmasında spektral analiz yöntemlerinden ve bu frekans bileşenlerinden yararlanılabilmektedir. Bu çalışmada zaman domaininden frekans domainine dönüşüm, Welch metodu yardımıyla herbir segmentin 'Spektral Güç Yoğunluğu (Power Spectral Density-PSD)' elde edilerek gerçekleştirilmiştir. Bu şekilde sinyalin değişik frekans değerlerine düşen enerji yoğunluğu hesaplanmış ve her segmentin gücü ve frekansı elde edilmiştir. Ardından herbir segment için Tablo 2'de görülen 15 özellik çıkarılmış ve $\boldsymbol{O}_{\boldsymbol{z}} \boldsymbol{e l l i k}$ Seti 1 oluşturulmuştur. Ayrıca Özellik Seti 1'e zaman domainindeki her segmentten Denklem 1, 2, 3 kullanılarak elde edilen 16., 17. ve 18. özellikler eklenmiştir.

Activity $=\operatorname{var}(\mathrm{X}(\mathrm{n}))$

Mobility $=\sqrt{\operatorname{Activity}\left(X^{\prime}(n)\right) / \operatorname{Activity}(\mathrm{X}(\mathrm{n}))}$ 
Denklemlerdeki $X(n)$ ve $X(n)$ ' sinyal ve sinyalin türevini ifade eder.

Tablo 2. Özellik Seti 1

\begin{tabular}{l|l}
\hline Özellik & Açıklama \\
\hline Ö1-5 & $\Delta, \Theta, \alpha, \sigma$ ve $>16 \mathrm{~Hz}$ Toplam Güç \\
\hline Ö6-10 & $\Delta, \Theta, \alpha, \sigma$ ve $>16 \mathrm{~Hz}$ Max. Güç \\
\hline Ö11-15 & $\Delta, \Theta, \alpha, \sigma$ ve $>16 \mathrm{~Hz}$ Min. Güç \\
\hline Ö16-18 & Activity, Mobility, Complexity \\
\hline
\end{tabular}

\subsubsection{Ayrık Dalgacık Dönüşümü ile Özellik Çıkarma}

EEG işaretleri statik olmayan yapıdadır. Dalgacık Dönüşümü statik olmayan sinyaller için diğer yöntemlere göre daha verimli sonuçlar üreten bir yöntemdir. Dalgacık Dönüşümünün en önemli avantajı, düşük frekanslar için geniş, yüksek frekanslar için dar olacak şekilde değişen pencere boyutlarının olmasıdır (Wong ve Leung, 1998). Dalgacık dönüşümleri tüm sinyal frekans-zaman bilgisini korurlar. $\mathrm{Bu}$ nedenle durağan olmayan gerçek doğal sinyallerinde bütün frekans aralıklarında optimum zaman-frekans çözünürlüğü sağlayarak geleneksel metotlardan daha iyi sonuçlar sağlar (Xu ve Ho, 2002).

'Ayrık Dalgacık Dönüşümü (Discrete Wavelet Transform-DWT)'nde her seviyede alçak frekansve yüksek frekans filtrelerinden geçirilerek yaklaşım ve ayrıntı bileşenleri elde edilir. Bir sonraki seviyede alçak frekans filtre çıkışına aynı işlemler uygulanır. Bu çalışmada sinyallere 3.seviye DWT uygulanarak sinyal frekans aralıklarına ayrılmıştır. DWT sayesinde ayrılan frekans aralıklarına göre, özel frekans bantları da kendiliğinden dalgacık üzerinde seçilir hale gelmiştir. DWT ile Tablo 3'deki özellikler çıkarılarak 21 özellikten oluşan $\boldsymbol{O}_{\text {zellik }}$ Seti 2 elde edilmiştir

Tablo 3. Özellik Seti 2

\begin{tabular}{l|l}
\hline Özellik & Açılama \\
\hline Ö1-5 & $\Delta, \Theta, \alpha, \sigma$ ve $>16 \mathrm{~Hz}$ Toplam Güç \\
\hline Ö6-10 & $\Delta, \Theta, \alpha, \sigma$ ve $>16 \mathrm{~Hz}$ Max. Güç \\
\hline Ö11-15 & $\Delta, \Theta, \alpha, \sigma$ ve $>16 \mathrm{~Hz}$ Min. Güç \\
\hline Ö16-20 & $\Delta, \Theta, \alpha, \sigma$ ve $>16 \mathrm{~Hz}$ Toplam Güç/Segment Gücü \\
\hline Ö21 & Alfa Gücü / Teta Gücü \\
\hline
\end{tabular}

Özellik Seti 1 ve Özellik Seti 2 'nin oluşturulmasının ardından, bu iki özellik seti birleştirilerek 39 özellikten oluşan Özellik Seti 3 elde edilmiştir. Bu sete ait ilk 19 özellik Özellik Seti 1'in özelliklerini içermektedir, Özellik Seti 3 'ün 20-39. özelliklerini ise Özellik Seti 2 oluşturmaktadır.

\subsection{Arousal Tespiti}

AASM'nin arousal tanımınca (AASM, 2012), bir arousal minimum 3 saniye olan bir süreci ifade etmektedir. Segmantasyon ile elde edilen segmentlerin arousal olarak etiketlenmesi için arousalların kaç saniyesinin o segmente denk geldiğinin bulunması gerekir. Bu doğrultuda arousalların en az 1 saniye ve üstü, 1 saniye üstü ve en az 2 saniye ve üstü olarak toplamda 3 farkl segment etiketleme çalışması yapılmıştır. Yapılan analiz çalışmaları sonucunda, bir segmentin arousal olarak etiketlenmesi işlemi en verimli sonuca ulaşılan mevcut arousalların 1 saniye üstünü barındıran segmentlerin arousal olarak etiketlenmesi şeklinde gerçekleştirilmiş̧ir.

Gerçekleştirilen işlemler sonucunda çalışmaya dahil edilen 5 hastaya ait 32397 normal segment ve 3367 arousal segment elde edilmiştir. Bu segmentler, segmentlerin yaklaşı \% 70 'i eğitim ve \%30'u test olacak şekilde rastgele ayrıştırılmıştır. Gerçekleştirilen sınıflama işlemleri için Weka (Witten ve ark., 1999) platformu kullanılmıştır. 


\section{6. Özellik Seçme}

Özellik Seti 1, Özellik Seti 2 ve Özellik Seti 3 için en iyi sınıflama performansına ulaşmak amacı ile Weka (Witten ve ark., 1999) platformunda bulunan Sarmal Alt Küme Değerlendirme (Wrapper Subset Evaluation-WSE) yöntemi ile özellik seçme işlemi gerçekleştirilmiştir. Her bir özellik setinin seçilen özellikleri ile optimum YSA ve RO algoritma parametreleri belirlenerek en başarılı sınıflandırma performansına ulaşılmaya çalışılmıştır.

YSA ve RO sınıflandırıcılarının WSE özellik seçme yöntemi ile kullanılmasıyla Özellik Seti1, Özellik Seti 2 ve Özellik Seti 3 için seçilen etkin özellikler sırasıyla Tablo 4 ve Tablo 5'de gösterilmiştir.

Tablo 4. WSE-YSA ile seçilen etkin özellikler

\begin{tabular}{l|l}
\hline Özellik Seti & Etkin Özellikler \\
\hline Set 1 & Ö1, Ö2, Ö3, Ö5, Ö6, Ö7, Ö8, Ö9, Ö11, Ö12, Ö14, Ö15, Ö17, Ö18 \\
\hline Set 2 & Ö4, Ö6, Ö8, Ö14, Ö19 \\
\hline Set 3 & Ö3, Ö22, Ö29, Ö37 \\
\hline
\end{tabular}

Tablo 5. WSE-RO ile seçilen etkin özellikler

\begin{tabular}{l|l}
\hline Özellik Seti & Etkin Özellikler \\
\hline Set 1 & Ö2, Ö3, Ö4, Ö5, Ö7, Ö8, Ö11, Ö12, Ö14, Ö15, Ö16, Ö17, Ö18 \\
\hline Set 2 & Ö1, Ö3, Ö4, Ö5, Ö8, Ö9, Ö10, Ö13, Ö14, Ö15, Ö16, Ö18, Ö19, Ö20 \\
\hline Set 3 & Ö2, Ö3, Ö4, Ö5, Ö7, Ö8, Ö11, Ö14, Ö16, Ö18, Ö28, Ö29, Ö31, Ö33, Ö38 \\
\hline
\end{tabular}

\section{Araştırma Sonuçları ve Tartışma}

Bu çalışmada, sınıflandırma başarısı, PSD ve DWT ile elde edilen 3 özellik setine WSE yöntemi ile özellik seçimi uygulanmasının ardından YSA ve RO sınıflandırıcıları kullanılarak elde edilen doğruluk, duyarlılık (recall) ve kesinlik (recision) performans ölçütleri ile değerlendirilmiştir. Eğitim için her iki sınıflandırıcı 10 kat çarpraz doğrulma yaklaşımı ile çalıştırılmıştır. Daha sonra, en yüksek doğruluğu sağlayan sınıflandırıcı parametrelerine göre test işlemleri gerçekleştirilmiştir. Tablo 6 ve Tablo 7 YSA kullanılması ile, Tablo 8 ve Tablo 9 ise RO sınıflandırıcı algoritmasının kullanılması ile elde edilen eğitim ve test sonuçlarını göstermektedir.

Tablo 6. Etkin özellikler ve YSA ile elde edilen ĕgitim sonuçları

\begin{tabular}{l|l|l|l}
\hline Özellik Seti & Duyarlılık & Kesinlik & Doğruluk \\
\hline Set1 & $60.1 \%$ & $93.6 \%$ & $96.03 \%$ \\
\hline Set2 & $53.2 \%$ & $100 \%$ & $95.78 \%$ \\
\hline Set3 & $53.8 \%$ & $98.6 \%$ & $95.77 \%$ \\
\hline
\end{tabular}

Tablo 7. Etkin özellikler ve YSA ile elde edilen test sonuçları

\begin{tabular}{l|l|l|l}
\hline Özellik Seti & Duyarlılık & Kesinlik & Doğruluk \\
\hline Set1 & $95.2 \%$ & $83.4 \%$ & $97.33 \%$ \\
\hline Set2 & $83.5 \%$ & $99.1 \%$ & $98.06 \%$ \\
\hline Set3 & $93.1 \%$ & $98.4 \%$ & $99.05 \%$ \\
\hline
\end{tabular}

Tablo 8. Etkin özellikler ve RO ile elde edilen eğitim sonuçları

\begin{tabular}{l|l|l|l}
\hline Özellik Seti & Duyarlılık & Kesinlik & Doğruluk \\
\hline Set1 & $67.9 \%$ & $91.5 \%$ & $96.54 \%$ \\
\hline Set2 & $54.8 \%$ & $98.8 \%$ & $95.87 \%$ \\
\hline Set3 & $65.7 \%$ & $92.9 \%$ & $96.46 \%$ \\
\hline
\end{tabular}


Tablo 9. Etkin özellikler ve RO ile elde edilen test sonuçları

\begin{tabular}{l|l|l|l}
\hline Özellik Seti & Duyarlılık & Kesinlik & Doğruluk \\
\hline Set1 & $87.2 \%$ & $72.6 \%$ & $94.85 \%$ \\
\hline Set2 & $74.5 \%$ & $99.1 \%$ & $97.05 \%$ \\
\hline Set3 & $87.2 \%$ & $76.5 \%$ & $95.55 \%$ \\
\hline
\end{tabular}

Gerçekleştirilen çalışmalar sonucunda en yüksek doğruluk oranı başarısına bakıldığında; YSA ve Özellik Seti 3'e ait PSD ile elde edilen $\alpha$-Toplam Gǚç ve DWT ile elde edilen $\sigma$-Toplam Gü̧̧, A-Min.Güç ve $\sigma$-Toplam Güç/Segment Gücü̈ özelliklerinin arousal tespiti üzerinde en fazla etkiye sahip olduğu söylenebilmektedir. Bu da özellik çıkarmada kullanılan PSD ve DWT yöntemlerinin her ikisinin de başarıda etkili olduğu, 5 adet özel frekans bantlarından en çok $\Delta$ (Delta), $\alpha$ (Alfa) ve $\sigma$ (Sigma) bantlarının başarıya etki ettiği ve YSA yönteminin sınıflandırmada RO’ya göre üstün performans sergilediği sonuçlarını ortaya koymaktadır.

\section{Sonuç}

$\mathrm{Bu}$ çalışmada uykuyu kesintiye uğratarak hastaların verimli bir gece uykusu almasına engel olan arousallar, tek kanallı EEG sinyalleri üzerinden sinyal işleme ve makine öğrenmesi yaklaşımları ile tespit edilmiştir. Sinyal işleme aşamasında filtreleme, normalizasyon, segmantasyon ve özellik çıkarma/seçme adımları uygulandıktan sonra, makine öğrenmesi aşamasında optimum parametre değerleri ile en başarılı sınıflandırıcı modelinin bulunması için YSA ve RO algoritmaları ile sınıflandırmalar yapılmıştır.

Sinıflandırma çalışmaları sonucunda görülmektedir ki, hem PSD hem de DWT ile elde edilen özelliklerin bulunduğu Özellik Seti 3'e ait 4 etkin özellik ve YSA yönteminin kullanılması ile test aşamasında en yüksek doğruluk oranı $\% 99.05$ olarak elde edilmiştir. Ayrıca bu sinıflandırma sonucunda duyarlılık ve kesinlik ölçütleri de sırasıyla \%93.1 ve \%98.4 olarak oldukça başarılı bulunmuştur. Elde edilen sonuçlara göre başka hiçbir PSG sinyal kaydına ihtiyaç duymadan, yalnızca tek kanallı EEG sinyallerine ait 4 etkin özellik ve YSA yöntemi ile arousal tespiti gerçekleştirilebileceği söylenebilir.

Her araştırma grubu kendi verilerini ve doğrulama kriterlerini kullandığı için, bu alandaki diğer çalışmalarla kıyaslamak için belirli bir standart yoktur (Uğur ve Erdamar, 2019). Ama bu alanda yapılan herbir çalışma ile her geçen gün daha da verimli çözümler elde edildiği görülmektedir.

\section{Kaynakça}

American Academy of Sleep Medicine. (2012). The AASM Manual for the Scoring of Sleep and Associated Events, Version v2.0. [Online]. Available: https://aasm.org/clinical-resources/scoring-manual/.

Demir, A., Ursavaş, A., Aslan, A. T., Gülbay, B., Çiftçi, B., Çuhadaroğlu, Ç., Keyf, F., Fırat, H., Yılmaz, M., Gerek, M., Köktürk, O., İtil, O., Karakoç, Ö., Başoğlu, Ö. K., Ersu, R., Ardıç, S., Öktem, S., Güven, S. F., \& Çiftçi, T. U. (2012). Türk Toraks Derneği Obstrüktif Uyku Apne Sendromu Tanı ve Tedavi Uzlaşı Raporu. Türk Toraks Dergisi, 13: 1-66.

Goldberger, A., Amaral, L., Glass, L., Hausdorff, J., Ivanov, P. C., Mark, R., Mietus, J. E., Moody, G. B., Peng, C. K., \& Stanley, H. E. (2000). PhysioBank, PhysioToolkit, and PhysioNet: Components of a new research resource for complex physiologic signals. Circulation [Online], 101: e215-e220. https://doi.org/10.1161/01.cir.101.23.e215.

Göğüş, F. Z., Tezel, G., Özşen, S., Küçç̋ktürk, S., Vatansev, H., \& Koca, Y. (2020). Identification of Apnea-Hypopnea Index Subgroups Based on Multifractal Detrended Fluctuation Analysis and Nasal Cannula Airflow Signals. Traitement du Signal, 37: 145-156. https://doi.org/10.18280/ts.370201.

Uğur, T. K., \& Erdamar, A. (2019). An efficient automatic arousals detection algorithm in single channel EEG. Computer Methods and Programs in Biomedicine, 137: 131-138. https://doi.org/10.1016/j.cmpb.2019.03.013.

Varela, F. I., Pereira, E. H., Estévez, D. Á., \& Bonillo, V. M. (2017). Combining Machine Learning Models for the Automatic Detection of EEG Arousals. Neurocomputing, 268: 100-108. https://doi.org/10.1016/j.neucom.2016.11.086.

Wallant, D. C., Mutoa, V., Gaggionia, G., Jaspara, M., Chellappaa, S. L., Meyera, C., Vandewallea, G., Maqueta, P., \& Phillips, C. (2016). Automatic artifacts and arousals detection in whole-night sleep EEG recordings. Journal of Neuroscience Methods, 258: 124-133. https://doi.org/10.1016/j.jneumeth.2015.11.005.

Witten, I. H., Frank, E., Trigg, L. E., Hall, M. A., Holmes, G., \& Cunningham, S. J. (1999) .Weka: Practical machine learning tools and techniques with Java implementations.

Wong, K., \& Leung, A. C. (1998). On-line successive synthesis of wavelet networks. Neural Processing Letters, 7: $91-100$. https://doi.org/10.1023/A:1009684412215.

$\mathrm{Xu}$, J. \& Ho, D. W. C. (2002). A basis selection algorithm for wavelet neural Networks. Neurocomputing, 48: 681-689. https://doi.org/10.1016/S0925-2312(01)00638-5. 